\title{
Can visual illustrations transform the patient information sheet for PET/MR neuroimaging studies into an engaging and interesting reading?
}

\author{
Giovanna Nordio $^{1}$ D $\cdot$ Gill Brown $^{2} \cdot$ Sagar Jilka $^{3,4} \cdot$ Alexander Hammers $^{5} \cdot$ Mitul A. Mehta $^{1} \cdot$ Anoushka Leslie $^{1}$. \\ Steve Williams ${ }^{1} \cdot$ Mattia Veronese $^{1,6}$
}

Received: 9 July 2021 / Accepted: 19 September 2021 / Published online: 5 October 2021

(c) The Author(s) 2021

Every research study with human participants requires a participant information sheet (PIS). This is an important document that aims to provide to the study participants, a full description of the study and all procedures, individual risks, and research benefits. But besides its informative use, the PIS also provides the legal basis for an individual to consent to participate and must be agreed and signed prior to participation.

PIS must be clear, concise, and easily understood by anyone, avoiding technical jargon as much as possible [1]. However, the complexity of modern medical research presents a challenge to explain in simple language, accessible by everyone. Neuroimaging studies are a representative case. They involve methodologies, such as magnetic resonance imaging (MRI) or nuclear medicine positron emission tomography (PET) that are technically complex. Nevertheless,

Giovanna Nordio

giovanna.nordio@kcl.ac.uk

Mattia Veronese

mattia.veronese@kcl.ac.uk

1 Department of Neuroimaging, Centre for Neuroimaging, Institute of Psychiatry, Psychology and Neuroscience (IoPPN), King's College London, PO89 De Crespigny Park, London SE5 8AF, UK

2 Freelance Graphic Designer, London, UK

3 Department of Psychology, Institute of Psychiatry, Psychology and Neuroscience, King's College London, London, UK

4 South London and Maudsley NHS Foundation Trust, London, UK

5 King's College London and Guy's and St Thomas' PET Centre, School of Biomedical Engineering and Imaging Sciences, King's College London, London, UK

6 Department of Information Engineering, University of Padua, Padua, Italy these methods are routinely used in medical research across spectrum of brain disorders to study aetiology, develop biomarkers and assess treatments. We aimed to tackle these challenges with informing participants of complex imaging research procedures, by developing a more visually appealing design procedure available to all researchers and working with local service users to understand the clarity and readability of the illustrated and fully written PIS versions.

To guarantee a collaborative individual's participation and a successful outcome from the study, it is paramount that the individual has a full understanding of the study before agreeing to take part [2]. Instead of having a PIS with only long and complex text, it would be beneficial to combine it with some visual elements to facilitate its understanding and effectively communicate the science. Like the safety instruction card of an airplane, visual elements could support a more efficient communication of PET/MR neuroimaging studies between researchers, clinicians and participants. We tested this approach, combining graphic design practise and science to create an illustrated participant information sheet. The project was conducted at the Centre for Neuroimaging Sciences, King's College London, in collaboration with a graphic designer with extensive experience in scientific visual communication, and with the patient and public involvement theme of the NIHR-Maudsley BRC for Mental Health. The aim was to create a modular illustrative participant information sheet for neuroimaging research studies conducted across King's College London imaging facilities. As a showcase for this project, the research study titled "Identifying neuroimaging markers for bipolar depression", conducted at the Institute of Psychology, Psychiatry and Neuroscience King's College London, was used. The study aims to understand the differences and similarities in the brain scans of people with bipolar disorder during an episode of depression, people with major depression disorder during an episode of depression and people with no history of significant mental health problems. 
The PIS of this study presented a similar structure to most of neuroimaging studies in agreement with regulation [2]: a first part describing the purpose of the investigations and what will happen if an individual decides to take part; the second part giving information about the conduct of the study.

For the realisation of the visual PIS, each section was divided in modules and for each module suitable visual elements were identified that could be used in the illustrations. These visual elements were then created using Adobe Illustrator software and, by fully utilising the 'Layer' functionality within Illustrator, were specifically designed to be easy to edit and adapt [3]. A range of images can then be exported from a single Illustrator file, simply by toggling the layer visibility. A small library of visual elements was created for use in the module illustrations and remain available for use in any future PIS. If no illustration was required for a module, the text was presented in the clearest way possible, using graphic elements such as tables, text boxes and rules to aid the reader. Colour-coding was used to differentiate the two sections, both on the pages of the PIS and in the colour palettes used for the illustrations. The modular structure used for the new visual PIS allows to easily readapt the document and the illustrations for different clinical studies, by simply including/removing specific modules related to the study of interest.

A survey was designed for the NIHR-Maudsley BRC Feasibility and Acceptability Support Team for Researchers (FAST-R) to compare the written-only and illustrative PIS in terms of clarity, understanding, readability and layout of the documents, and the role of the illustrations. The FAST-R is a service comprising individuals with extensive expertise of mental health services as a service user or carer. Unanimously, all the respondents agreed that the inclusion of visual illustrations in the PIS significantly helps their understanding of the document: in a scale from 0 (low) to 5 (very high) the average scores were 5 for clarity, 4.4 for impact of illustrations, and 4.5 for readability and layout. Similarly, feedback from both the NIHR-Maudsley BRC Service User Advisory Group and the Young People's Mental Health Advisory Group (YPMHAG) collected on a representative showcase for a neuroimaging study strongly support the use of visual components within PIS, as it facilitates the reading and comprehension of the research study, especially for people with literacy problems. In this case, a more friendly layout was used, which combined text, illustrations, and the use of colours (Fig. 1). The selection of the colours and the font size used was chosen to improve universal accessibility
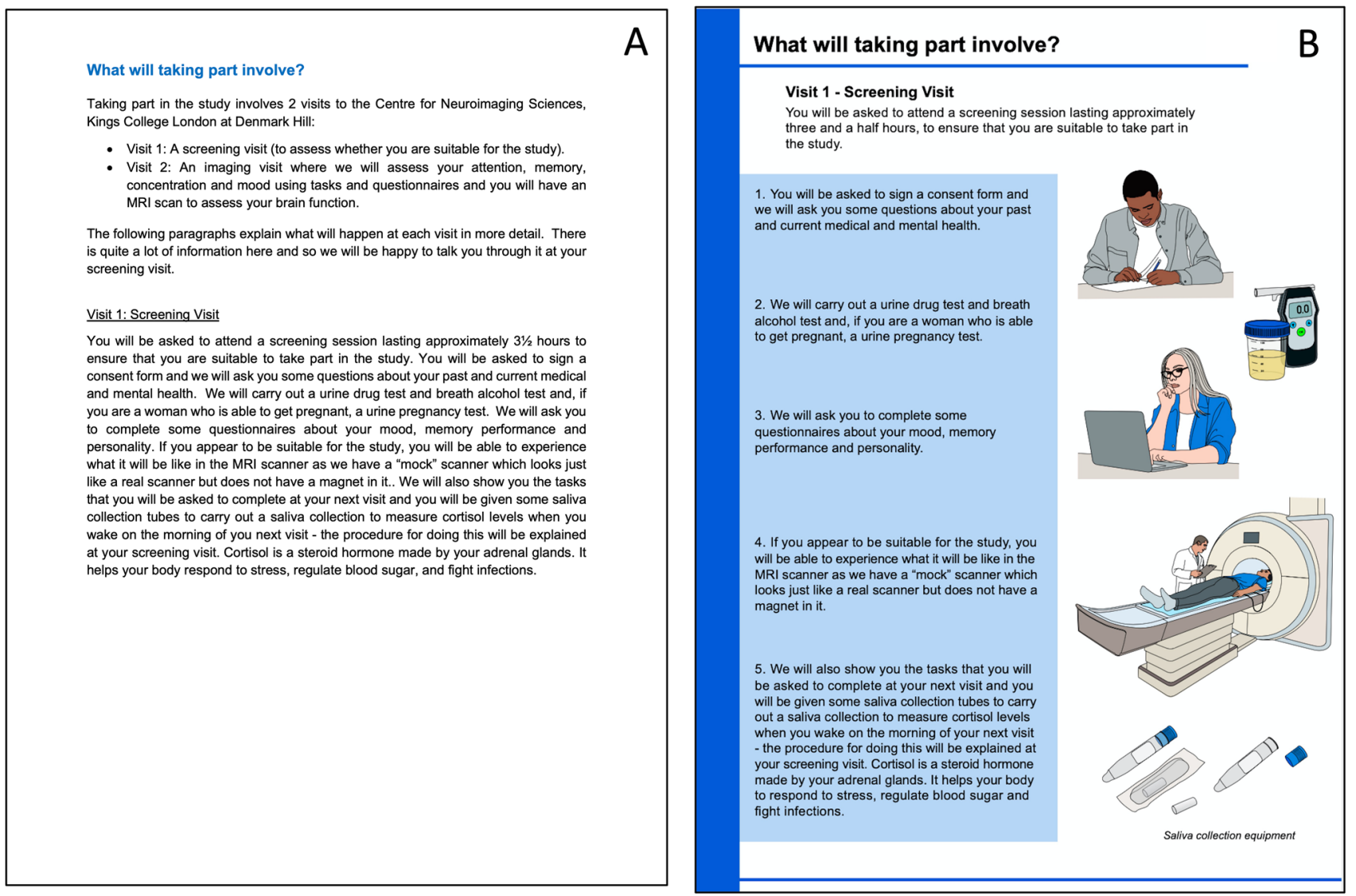

Fig. 1 Section titled "What will taking part involve?" for respectively the written-only (A) and illustrative (B) patient information sheet 

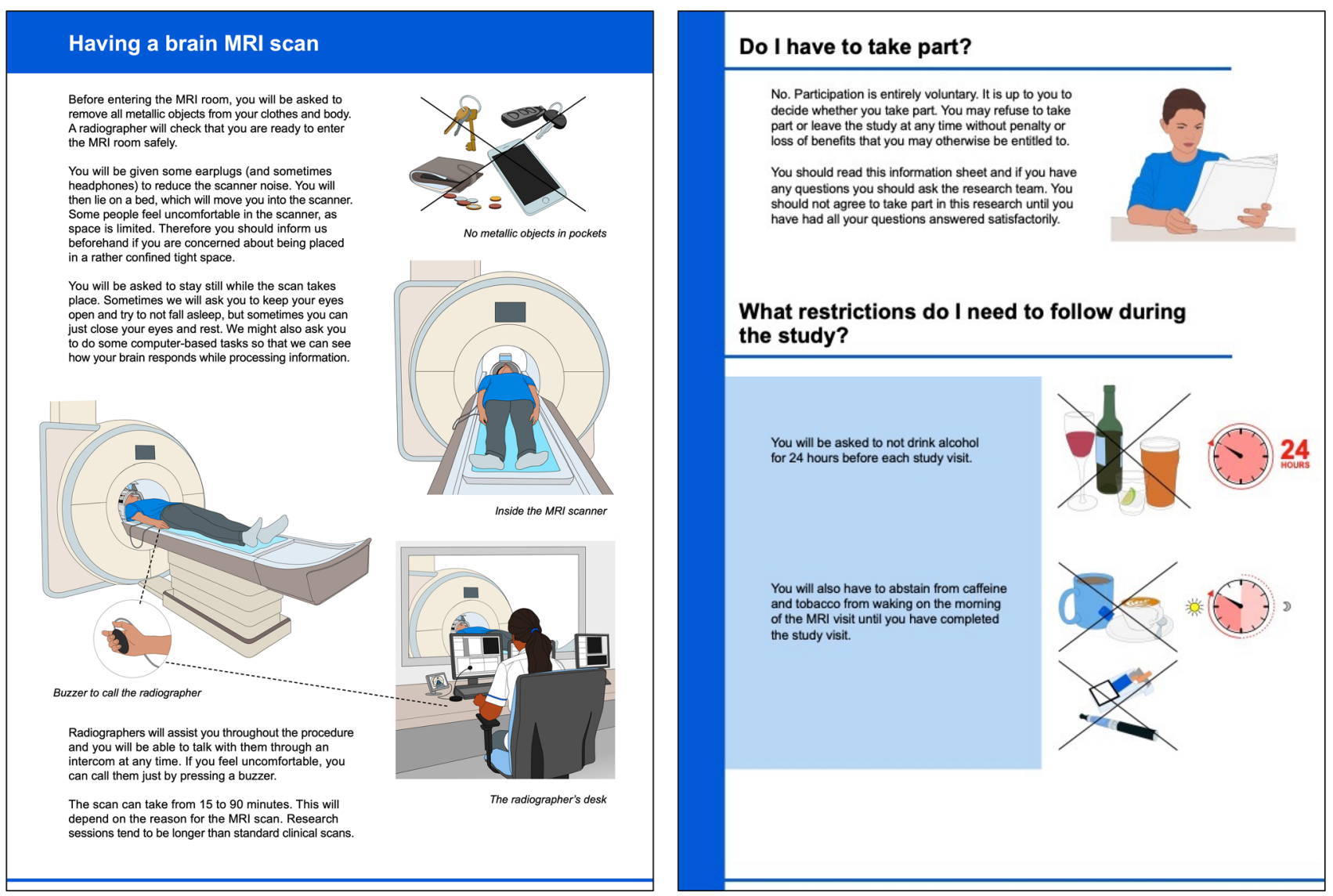

Fig. 2 Section of the illustrative PIS about the MRI scan

of the PIS. The illustrations of patients and staff was as much representative as possible of the local client and staff demographics. Illustrations help to simplify the explanation of the MRI scan, making the reading easier and more informative (Fig. 2).

While these results should be confirmed in a large prospective study, they confirm how a graphical and simplified appearance of an illustrated participant information sheet helps to better communicate the research and to ensure a more informed decision from the participants. The use of visual components can transform the participant information sheet of any PET/MRI study from a mandatory and legal document into an interesting and engaging reading for researchers, clinicians and patients while retaining adherence to core requirements. With the right training and software any scientist can build their own illustrations and contribute to making the communication of science more appealing.

Acknowledgements We want to thank Claudia Cecconi and Paul Stokes for contributing to the original PIS.
Resources The illustrative PIS can be downloaded from the neuroscience graphic design webpage.

Funding This study was funded by the Wellcome EPSRC Centre for Medical Engineering (CME) Public Engagement Grant Scheme. This paper represents independent research funded by the National Institute for Health Research (NIHR) Maudsley Biomedical Research Centre at South London and Maudsley NHS Foundation Trust and King's College London. The views expressed are those of the authors and not necessarily those of the NHS, the NIHR or the Department of Health and Social Care.

\section{Declarations}

Conflict of interest There is no conflict of interest in relation to this article.

Open Access This article is licensed under a Creative Commons Attribution 4.0 International License, which permits use, sharing, adaptation, distribution and reproduction in any medium or format, as long as you give appropriate credit to the original author(s) and the source, provide a link to the Creative Commons licence, and indicate if changes were made. The images or other third party material in this article are included in the article's Creative Commons licence, unless indicated otherwise in a credit line to the material. If material is not included in the article's Creative Commons licence and your intended use is not 
permitted by statutory regulation or exceeds the permitted use, you will need to obtain permission directly from the copyright holder. To view a copy of this licence, visit http://creativecommons.org/licenses/by/4.0/.

\section{References}

1. Alexa-Stratulat T, Neagu M, Neagu A-I, Alexa ID, Ioan BG (2018) Consent for participating in clinical trials-is it really informed? Dev World Bioeth 18:299-306
2. Health Research Authority Consent and participant information guidance. http://www.hra-decisiontools.org.uk/consent/index.html

3. Brown G (2019) Visual elements and visual paradigms: re-thinking scientific conceptual figures through graphic design. University of the Arts London

Publisher's Note Springer Nature remains neutral with regard to jurisdictional claims in published maps and institutional affiliations. 